\title{
Semi-Rational Screening of Probiotics from the Fecal Flora of Healthy Adults against DSS-Induced Colitis Mice by Enhancing Anti-Inflammatory Activity and Modulating the Gut Microbiota
}

Weiwei Wang ${ }^{1 \dagger}$, Wentao Xing ${ }^{1+}$, Sichen Wei ${ }^{1 *}$, Qiaoying Gao ${ }^{2}$, Xinliang Wei ${ }^{1}$, Liang Shi ${ }^{3}$, Yu Kong ${ }^{1}$, and $\mathrm{Zhenhua} \mathrm{Su}^{1}$

${ }^{1}$ Department of Gastroenterology, Cangzhou Central Hospital, No. 16 Xinhua West Road, Cangzhou, Hebei 061001, P.R. China

${ }^{2}$ Tianjin Institute of Acute Abdominal Diseases, Nankai Hospital, Nankai District, Tianjin, 300100, P.R. China

${ }^{3}$ Endoscopy Room, Cangzhou Central Hospital, No. 16 Xinhua West Road, Cangzhou, Hebei 061001, P.R. China

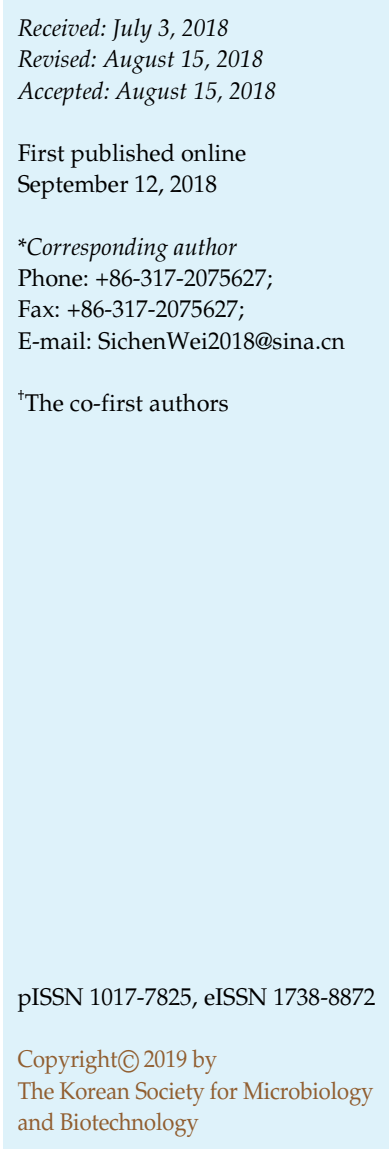

\begin{abstract}
Ulcerative colitis (UC), a chronic inflammatory bowel disease, substantially impacts patients' health-related quality of life. In this study, an effective strategy for discovering high-efficiency probiotics has been developed. First, in order to survive in the conditions of the stomach and intestine, high bile salt-resistant and strong acid-resistant strains were screened out from the fecal flora of healthy adults. Next, the probiotic candidates were rescreened by examining the induction ability of IL-10 (anti-inflammatory factor) production in dextran sodium sulfate (DSS)-induced colitis mice, and Lactobacillus sakei 07 (L07) was identified and selected as probiotic P. In the end, fourteen bifidobacterium strains isolated from stools of healthy males were examined for their antimicrobial activity. Bifidobacterium bifidum B10 (73.75\% inhibition rate) was selected as probiotic B. Moreover, the colonic IL- 6 and TNF- $\alpha$ expression of the DSSinduced colitis mice treated with L. sakei 07 (L07) - B. bifidum B10 combination (PB) significantly decreased and the IL-10 expression was up-regulated by PB compared to the DSS group. Furthermore, Bacteroidetes and Actinobacteria decreased and Firmicutes increased in the DSS group mice, significantly. More interestingly, the intestinal flora biodiversity of DSS colitis mice was increased by PB. Of those, the level of B. bifidum increased significantly. The Bacteriodetes/Firmicutes (B/F) ratio increased, and the concentration of homocysteine and LPS in plasma was down-regulated by PB in the DSS-induced colitis mice. Upon administration of $\mathrm{PB}$, the intestinal permeability of the the DSS-induced colitis mice was decreased by approximately 2.01-fold. This method is expected to be used in high-throughput screening of the probiotics against colitis. In addition, the L. sakei 07 - B. bifidum B10 combination holds potential in UC remission by immunomodulatory and gut microbiota modulation.
\end{abstract}

Keywords: Probiotics, ulcerative colitis, anti-inflammatory, gut microbiota, immunomodulatory, homocysteine, intestinal permeability

\section{Introduction}

Inflammatory bowel disease (IBD) includes ulcerative colitis (UC) and Crohn's disease (CD) [1]. Ulcerative colitis (UC), a chronic or long lasting IBD, is difficult to cure due to its rising incidence in recent decades [2]. To our best knowledge, UC causes many sporadic symptoms, including abdominal pain, diarrhea, and bloody mucopurulent stool [3], which is often accompanied by an increase in intestinal tract disorders [3] and inflammatory mediators [4].

Gut microbiota can be divided into three categories: (A) Symbiotic bacteria, including Bacteroides, Bifidobacterium bifidum, Lactobacillus, etc.; (B) Opportunistic pathogens, including Enterococcus, Enterobacter, etc.; (C) Pathogenic 
bacteria. The imbalance of gut microbiota is associated with an interaction among inflammation mechanisms, host defense modulation, oxidative stress, and alterations in bacterial-derived metabolism [5]. Colitis can be ameliorated by precision editing of the gut microbiota [6]. Moreover, the generation of inflammation is promoted by a large number of pro-inflammatory factors (such as IL-6, and TNF- $\alpha$ ). Intestinal permeability can be worsened by intestinal pathogens and an associated immune response which can then result in disrupted epithelial barrier function. Due to the destruction of the intestinal mucosal integrity, the barrier function of the prosthetic layer weakens, resulting in the intestinal immune system's loss of tolerance to intestinal bacteria.

Inducing remission and preventing recurrence were the goal of UC treatment [7]. Nowadays, the major drugs used in UC management are corticosteroids, 5-aminosalicylates, immunomodulators and biologics. However, the severe adverse side effects are also very obvious, such as nephrotoxicity, fever, rash, drug hypersensitivity, hepatitis, pancreatitis, lymphadenopathy, abdominal pain, nausea, vomiting, diarrhea exacerbation, and myalgia [8]. Due to dissatisfaction with these medications, probiotics, which were only discovered within recent years, are now seen as a potential complementary and alternative therapy for the treatment of UC and related illness.

Probiotics can enhance the intestinal barrier, regulate the immune system and improve health performance [9]. Probiotics have been used in UC remission via barrier function modulation, which involves the competition of nutrients and the production of bacteriocin or antibacterial proteins. It has been reported that probiotics, such as Lactococcus lactis subsp. lactis [10], L. curvatus [11], and L. plantarum [12] ameliorate colitis by regulating the expression of IL-10, TNF- $\alpha$, and IL-6. Strategies for increasing the abundance of gut bacteria have targeted the production of lactic acid bacteria, especially lactobacilli and bifidobacteria $[13,14]$.

Fecal microbiota transplantation (FMT), also called fecal bacteriotherapy, has been used for 50 years for treatment of Clostridium difcile-associated diarrhea and pseudomembranous colitis with great success and few adverse effects [15]. In our present work, probiotics were screened from fecal flora of healthy adults, and the probiotics combinations (L. sakei- B. bifidum) have been designed for remission of the DSS-induced colitis disease. Probiotics hold potential for application against UC via gut microbiota modulation and anti-inflammatory properties.

\section{Materials and Methods}

\section{Screening of Strains}

Lactic acid bacterial strains were isolated from fecal microbiota of healthy adults. To ensure general health, the criteria for the healthy adult volunteers had to meet the following standards: 1) be free of known metabolic and gastrointestinal diseases, with no history of metabolic or gastrointestinal diseases; 2) avoid taking medications or alcohol that would impact gut function; 3) be willing to complete all necessary study questionnaires and to donate stool specimens as required; 4) to voluntarily sign a written informed consent form before participation in the study. Lactic acid bacteria were isolated on MRS agar ( $\mathrm{pH}$ 5.6) incubated at $30^{\circ} \mathrm{C}$ for $72 \mathrm{~h}$. Acid-resistance of lactic acid bacteria colonies isolated from the MRS agar was screened by culturing on MRS agar ( $\mathrm{pH}$ 3.0). The bile salt tolerance of the colonies was screened by culturing on MRS agar containing $0.30 \%$ ox bile salts at $37^{\circ} \mathrm{C}$. Bifidobacteria were selective cultured anaerobically in $\mathrm{MRS} \mathrm{cm}$ (MRS medium supplemented with $0.5 \mathrm{~g} / 1 \mathrm{~L}$-cysteine and $50 \mathrm{mg} / 1$ mupirocin) at $37^{\circ} \mathrm{C}$ according to the method of Ferraris L [8]. In order to obtain a monoclonal strain, a single colony was repeatedly restreaked on MRScm agar or MRS agar. Chromosomal DNA was isolated and the 16S rRNA gene was amplified by PCR [3]. The strains were identified by DNA sequencing (Sango Biotech, China) according to the method of Tyagi, N. et al. [16].

\section{Animals}

C57BL/6J mice (seven weeks old, male) were purchased from Super-B\&K Laboratory Animal Corp., Ltd. (China). The mice were kept at $22^{\circ} \mathrm{C}$ under a 12-h light/dark cycle, supplied with sufficient water and standard rodent diet, and acclimatized to laboratory conditions for 7 days before conducting experiments. Mice were divided into 4 groups $(n=12)$ with different treatments. Control Group: normal chow diet. DSS Group: normal chow diet and drinking water with $3 \%$ acute dextran sulfate sodium (DSS) for one week (7 days). DSS+P Group: normal chow diet and drinking water with 3\% DSS for 7 days, $2.0 \mathrm{ml}$ Lactobacillus sakei 07 (L07) $\left(1 \times 10^{10} \mathrm{CFU} / \mathrm{ml}\right)$ was administered orally ( 3 times/day) from the 4 rd to 8th day. DSS+PB Group: normal chow diet and drinking water with $3 \%$ DSS for 7 days, $2.0 \mathrm{ml}$ mixture of Lactobacillus sakei 07 (L07) $\left(1 \times 10^{10} \mathrm{CFU} / \mathrm{ml}\right)$ and Bifidobacterium bifidum B10 $\left(2 \times 10^{10} \mathrm{CFU} / \mathrm{ml}\right)$ were administered orally (3 times/day) from the 4 th to 8 th day. Afterwards, the mice were sacrificed on the 9 th day. Serum and colons were collected for follow-up experiment. Colonic tissue was homogenized and centrifuged at $4^{\circ} \mathrm{C}$ $(12,000 \mathrm{~g} \times 15 \mathrm{~min})$, and subsequently the supernatants were used to determine cytokines by ELISA kit (R\&D Systems, USA) according to the manufacturer's instructions. Endotoxin levels (lipopolysaccharides, LPS) were measured by using a limulus amebocyte lysate assay (LAL) endotoxin assay kit (USA). The levels of homocysteine (Hcy) in plasma and colon mucosa were measured by high-performance liquid chromatography-fluorescence 
detection according to the report of Chen $\mathrm{M}$ et al. [17]. This study was approved by the Ethics Committee of Cangzhou Central Hospital (approval No. 2017-063) (Cangzhou, China). All animal care and experimental protocols were approved by the Animal Care and Research Committee of Cangzhou Central Hospital.

\section{Analyses of Hemoglobin Concentration in Blood and DAI}

Hemoglobin concentration was determined according to the method of HiCN [18]. The disease activity indices (DAI) composed of three parameters (weight loss, stool consistency, and bloody stools) were calculated to assess severity of colitis according to the report of Sang LX [19]. Briefly, the disease activity index consisted of scoring for stool consistency (0-3) body weight loss (0-3), and visual observation of blood in feces $(0-4)$.

\section{Quantitative Real-Time Polymerase Chain Reaction (qrtPCR)}

A fresh colon sample was used for total RNA extraction from with TRIZOL reagent (Invitrogen Life Technologies Co Ltd., USA) following the manufacturer's protocol. The relative mRNA expression of cytokines was determined by quantitative real-time polymerase chain reaction (qrt-PCR) with optimal concentrations of primers and probes. Mouse TGF- $\beta$ primer (forward $5^{\prime}$-ACGAC ATGATAGTCACTGACAACA and reverse $5^{\prime}$-TTGGGGTCA TGGCAAACTGTCTC); TNF- $\alpha$ primer (forward 5 '-AGATGTGGA ACTGGCAGAGG; antisense 5'-CACGAGCAGGAATGAGAAGAG), IL-10 primer pair (forward 5'-ACTGCTATGTTGCCTGCTCTT; antisense $5^{\prime}$-GGTCTGGCTGACTGGGAAGT) and IL-6 primer pair (sense 5'-TAGTCCTTCCTACCCCAATTTCC; antisense 5' - TTGGTC CTTAGCCACTCCTTC) were used as the primers or probes. Mouse GAPDH primers (forward: 5'-GTATGACTCCACTCACGGCAAA and reverse, 5'-GGTCTCGCTCCTGGAAGATG) were used as internal controls. The thermal cycler conditions were 30 cycles of $95^{\circ} \mathrm{C}$ for $15 \mathrm{sec}, 58^{\circ} \mathrm{C}$ for $20 \mathrm{sec}$, and $72^{\circ} \mathrm{C}$ for $30 \mathrm{sec}$. All samples were run in duplicate. Relative quantity or fold change in gene expression of target genes relative to the house keeping gene $2^{-\Delta \Delta C t}$, where $\Delta \Delta \mathrm{C}_{\mathrm{t}}=\Delta \mathrm{C}_{\mathrm{t}}$ control $-\Delta \mathrm{C}_{\mathrm{t}}$ treated.

\section{Analysis of Intestinal Bacteria}

The composition of the bacteria present in colonic contents of the mice was detected by quantitative PCR according to the method of Long T et al. [20]. Briefly, microbial DNA was extracted from fecal contents with the E.Z.N.A. Stool DNA Kit (Omega Biotek, USA). Extracted bacterial DNA was submitted to quantitative PCR. Moreover, the abundance of five representative bacteria in fecal samples was based on the detection of $16 \mathrm{~S}$ rRNA genes. PCR products were extracted from $2 \%$ agarose gels and purified with the AxyPrep DNA Gel Extraction Kit (Axygen Biosciences, USA). The primer sequences were as follows: Escherichia coli F:(5'CATGCCGCGTGTATGAAGAA) and R: (5'-CGGGTAACGTCA ATGAGCAAA), Enterococcus spp. F: (5'-GACGAAAGTCTGAC CGAGCA) and R: (5'-TTAGCCGTGGCTTTCTGGTT), Lactobacillus F: (5'-AGCAGTAGGGAATCTTCCA) and R: (5'-CACCGCTAC ACATGGAG), Bifidobacteria F: (5'-GCGTGCTTAACACATGCA
AGTC) and R: (5'-CACCCGTTTCCAGGAGCTATT), Bacteroides F: (5'-GAGGAAGGTCCCCCACATTG) and R: (5'-ACCCATAGG GCAGTCATCCT). Bacteroidetes F: (5'-AACAGGATTAGATACCCT) and R: (5'-GGTAAGGTTCCTCGCGTAT). Firmicutes F: (5'-TGA AACTYAAAGGAATTGACG) and R: (5'-ACCATGCACCACCTGTC). Actinobacteria F: (5'-TACGGCCGCAAGGCTA) and R: (5'-TCC CCACCTTCCTC). Proteobacteria F: (5'-TCGTCAGCTCGTGTY GTGA) and R: (5'-CGTAAGGGCCATGATG).

\section{Measurement of the Colonic Mucosal Permeability In Vitro}

The colonic mucosal permeability was examined by Evans Blue (EB) assay [21]. A small bowel was infused with $0.3 \mathrm{ml}$ EB solution. After washing with normal saline, the small bowel was dried for $24 \mathrm{~h}$, and incubated with $1 \mathrm{ml}$ of formamide for $24 \mathrm{~h}$. After centrifuging, the supernatant was collected and examined under an ultraviolet spectrophotometer at a wavelength of $620 \mathrm{~nm}$.

\section{Results}

\section{Probiotic Screening}

FMT has been used for treatment of Clostridium difcileassociated diarrhea and pseudomembranous colitis [15], highlighted by a disease improvement or resolution rate of $83 \%-92 \%[22,23]$. In addition, strains as a probiotic should survive in the conditions of the stomach and the bile salts. Therefore, high-bile salt and strong acid-resistant strains were screened from the fecal flora of healthy adults. A total of 76 strains were obtained from the fecal flora of healthy adults (the donors). Afterwards, the tolerance of strains in acid conditions ( $\mathrm{pH} 3.0$ ) and $0.3 \%$ bile salts was investigated, and the strains indicated in red boxes were selected as the candidates (Fig. 1A). IL-10 is an anti-inflammatory cytokine, mainly secreted by Th2 cells and mononuclear macrophages, which can inhibit the secretion of pro-inflammatory cytokines [24]. Thus, the effect of different candidates on IL-10 production by stimulation of splenic lymphocytes in vitro has been investigated. Of those, strain 07 and strain 34 showed higher capacity on IL-10 production by stimulating the spleen cells (Fig. 1B). Strain 07 and strain 34 were identified as Lactobacillus sakei and Pediococcus acidilactici by DNA sequencing. In addition, Lactobacillus are widely used as effective and safe probiotics. Therefore, Lactobacillus sakei 07 (L07) was selected as probiotic P.

Bifidobacterium spp. has been used as a preferred drug in UC patients [25]. Moreover, we found that the abundance of Bifidobacterium was lower in DSS-induced colitis mice comparing to the healthy mice (Fig. 4D). Therefore, fourteen Bifidobacterium strains isolated from the fecal flora of healthy adults were examined for their antimicrobial activity with E. coli as an indicator, which were isolated 
A
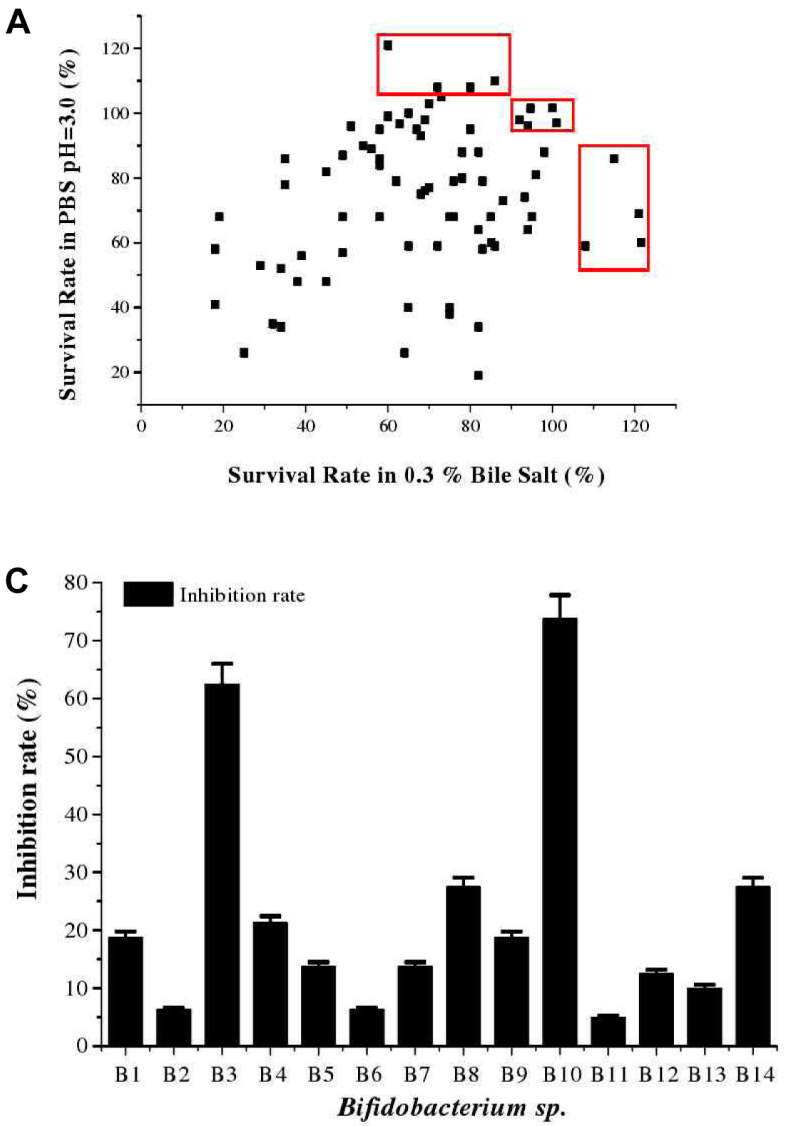

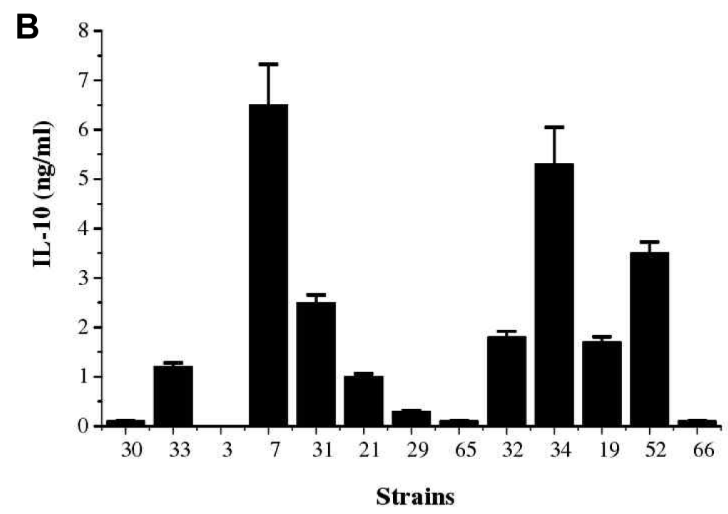

D

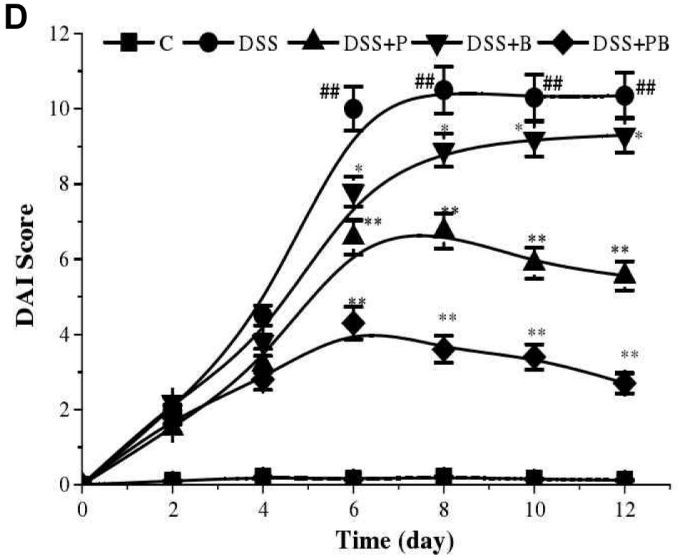

Fig. 1. Strains screened from the fecal flora of healthy adults (A). Survival rate $\%$ (PBS, $\mathrm{pH}=3.0$ ) $=\mathrm{N}_{4} / \mathrm{N}_{0} \times 100 \%$; Survival rate $\%$ $(0.3 \%$ bile salt $)=\mathrm{N}_{4 \mathrm{~b}} / \mathrm{N}_{0 \mathrm{~b}} \times 100 \% . \mathrm{N}_{4}$, Number of lactic acid bacteria $(\log \mathrm{CFU} / \mathrm{ml})$ after incubation for $4 \mathrm{~h}$ at $37^{\circ} \mathrm{C} ; \mathrm{N}_{0}, \mathrm{Number}$ of lactic acid bacteria (log CFU/ml) after incubation for $0 \mathrm{~h}$ at $37^{\circ} \mathrm{C}$ in MRS broth $(\mathrm{pH}=3.0) ; \mathrm{N}_{4 \mathrm{~b}}$, Number of lactic acid bacteria (log $\mathrm{CFU} / \mathrm{ml}$ ) after incubation for $4 \mathrm{~h}$ at $37^{\circ} \mathrm{C}$ in MRS broth containing $0.30 \%$ bile salts $(\mathrm{pH}=6.0) ; \mathrm{N}_{0 \mathrm{~b}}$, Number of lactic acid bacteria $(\log \mathrm{CFU} / \mathrm{ml})$ after incubation for $0 \mathrm{~h}$ at $37^{\circ} \mathrm{C}$ in MRS broth containing $0.30 \%$ bile salts ( $\left.\mathrm{pH}=6.0\right)$. The concentrations of the candidates were $1 \times 10^{6} \mathrm{CFU} / \mathrm{ml}$. The concentrations of IL-10 were measured by ELISA. Screening of the functional Bifidobacterium strains on inhibition of E. coli isolated from bloody stools of DSS-induced colitis mice (C). The E. coli $\left(1 \times 10^{8} \mathrm{CFU} / \mathrm{ml}\right)$ were cocultured with Bifidobacterium for three hours at $37^{\circ} \mathrm{C}$. (D) Disease activity index, a composite measure of weight loss, stool consistency and blood in stool. Data presented indicate the mean \pm SD. \#\#p $<0.01$ vs C group, ${ }^{*} p<0.05$ and ${ }^{* *} p<0.01$ vs DSS group.

from bloody stools of DSS-induced colitis mice (Fig. 1C). The inhibition rate of the E. coli was investigated and the results showed that Bifidobacterium bifidum B10 having high performance $(73.75 \%$ inhibition rate) and therefore it was selected as probiotic B. In order to assess the severity of DSS-induced colitis, the Disease Activity Index (DAI) score and blood hemoglobin (associated with severity of UC) were also investigated. The results showed that probiotic B (Bifidobacterium bifidum B10) alone failed to increase the blood haemoglobin level in DSS-induced colitis, by comparison with probiotic $\mathrm{PB}$ and probiotic $\mathrm{P}$ groups
(Table 1). The DAI score of probiotic B is higher than that of probiotic PB and probiotic P group (Fig. 1D). The effect of probiotic B (Bifidobacterium bifidum B10) treatment alone on ameliorating DSS-induced colitis is not significant. Therefore, probiotic $\mathrm{PB}$ and probiotic $\mathrm{P}$ groups were selected for further study.

\section{Effects of Probiotic PB on DSS-Induced Colitis Mice}

The function of probiotic PB in DSS-induced colitis mice was examined in vivo. C57BL/6 J mice were pre-treated with probiotics $\mathrm{P}$ or $\mathrm{PB}$ for four days and then administered DSS 
Table 1. Effect of probiotics on blood haemoglobin.

\begin{tabular}{lc}
\hline \multicolumn{1}{c}{ Group } & Blood haemoglobin $(\mathrm{g} / \mathrm{dL})$ \\
\hline C & $13.02 \pm 0.07$ \\
DSS & $9.65 \pm 0.28^{\#}$ \\
Probiotic P & $10.86 \pm 0.29$ \\
Probiotic B & $9.88 \pm 0.26$ \\
Probiotic PB & $11.87 \pm 0.11^{*}$ \\
Mesalazine group & $12.74 \pm 0.32^{*}$ \\
\hline
\end{tabular}

Note: $\mathrm{C}$ group: normal diet $+350 \mu$ sterile water (intragastric administration); DSS group: $3 \%$ DSS model $+350 \mu$ sterile water; Positive control group: $3 \%$ DSS model $+350 \mathrm{ul} 20 \mathrm{mg} / \mathrm{ml}$ mesalazine; Probiotic P group: 3\% DSS model + $350 \mathrm{ul}$ $1 \times 10^{10} \mathrm{CFU} / \mathrm{ml}$ Lactobacillus sakei 07 (L07); Probiotic B group: 3\% DSS model + $350 \mathrm{ul} 1 \times 10^{10} \mathrm{CFU} / \mathrm{ml}$ Bifidobacterium bifidum B10; Probiotic PB group: 3\% DSS model $+175 \mathrm{ul} 1 \times 10^{10} \mathrm{CFU} / \mathrm{ml}$ Lactobacillus sakei 07 (L07) $+175 \mathrm{ul} 1 \times 10^{10} \mathrm{CFU} / \mathrm{ml}$ Bifidobacterium bifidum B10. Blood haemoglobin is expressed as the mean \pm SD. $\# p<0.05$ vs the normal group. ${ }^{*} p<0.05$ vs the DSS group.

in drinking water for another four days to induce colitis. Treatment with probiotic PB was continued after DSS administration. The result was shown in Fig. 2. Comparing to the control group, the colon length of DSS group mice was significantly decreased and colon weight/colon length were increased (Fig. 2A). The DSS group had a significantly higher colon weight/length ratio $(63 \pm 0.92 \mathrm{mg} / \mathrm{cm})$ compared to the other groups. Moreover, it was observed that the colon weight/length ratio of the DSS-induced mice could be reduced by probiotic PB. Also, the role of probiotic $\mathrm{P}$ was enhanced by Bifidobacterium bifidum B10 (probiotic B). In addition, the colon weight/length ratio of the control group $(3.8 \pm 0.31 \mathrm{mg} / \mathrm{cm})$ was basically the same as that of the DSS-treated group which received probiotic PB treatment $(4.1 \pm 0.25 \mathrm{mg} / \mathrm{cm})$ (Fig. 2B).

Furthermore, the effect of probiotic PB on mRNA expression of cytokines in DSS-induced colitis mice has been investigated. The mRNA expression of IL-10 and TGF- $\beta$ was down-regulated in DSS-induced mice as compared to control group. The TGF- $\beta$ and IL-10 expression of the DSS group was up-regulated by administration of probiotic $\mathrm{P}$ or $\mathrm{PB}$, however, the difference between probiotic $\mathrm{P}$ and probiotic $\mathrm{PB}$ is not significant (Figs. 3A and $3 \mathrm{~B})$. The results demonstrated that mRNA levels of IL-6 and TNF- $\alpha$ were elevated in DSS group compared with the control group. Colonic IL-6 and TNF- $\alpha$ expression of DSSinduced mice was significantly reduced after administration of probiotic P or PB (Figs. 3C and 3D).

\section{Effect of Probiotic PB on the Bacterial Communities of DSS-Induced Colitis Mice}

The relative abundances of different phyla in feces samples were shown in Fig. 4 . The high-abundant ( $>1 \%$ of community) were investigated, including Firmicutes, Bacteroidetes, Proteobacteria, Actinobacteria. The abundance of Firmicutes was increased and the abundance of Bacteroidetes and Actinobacteria was decreased in DSS group compared with the $C$ group (the control, healthy mice), significantly (Fig. 4A).

In addition, the Bacteriodetes / Firmicutes (B/F) ratio was decreased in DSS-induced mice when compared to healthy mice (C group) (Fig. 4B), while the B/F ratio of the DSSinduced colitis mice increased after oral administration of probiotic $\mathrm{P}$. More interestingly, the ability of probiotic $\mathrm{P}$
A

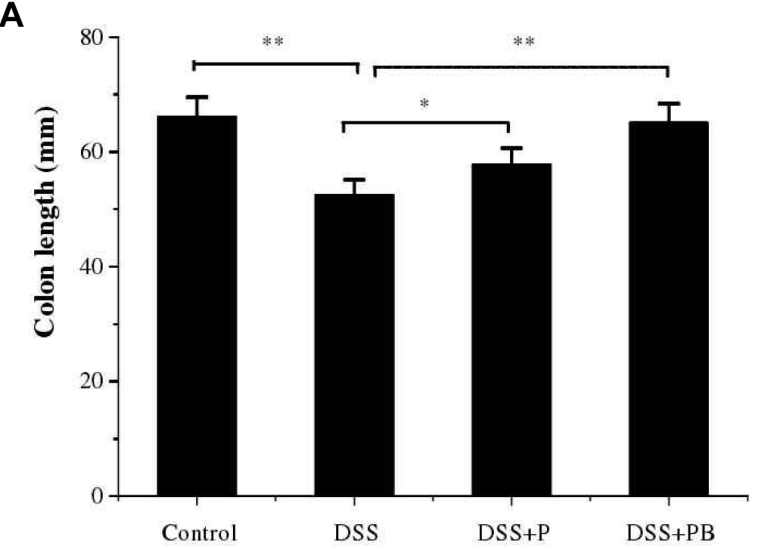

B

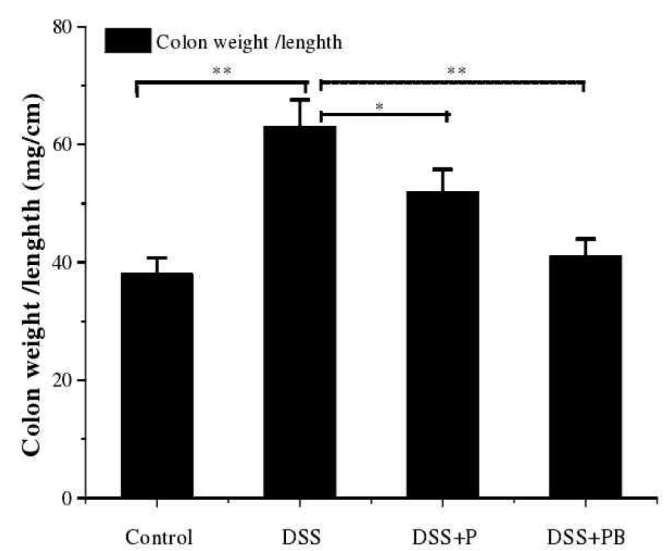

Fig. 2. PB ameliorated colonic injury in dextran sodium sulfate (DSS)-induced chronic colitis mice. (A) Colon length. (B) Colon weight/Colon length $(\mathrm{mg} / \mathrm{cm}$ ). Abbreviation: C, Control group; DSS, DSS group; DSS+P, DSS + Lactobacillus sakei 07 (L07); (4) DSS + PB, DSS + Lactobacillus sakei 07 (L07) + Bifidobacterium bifidum B10. Values were expressed as the mean $\pm \mathrm{SD},{ }^{*} p<0.05,{ }^{* *} p<$ 0.01 as conducted. 


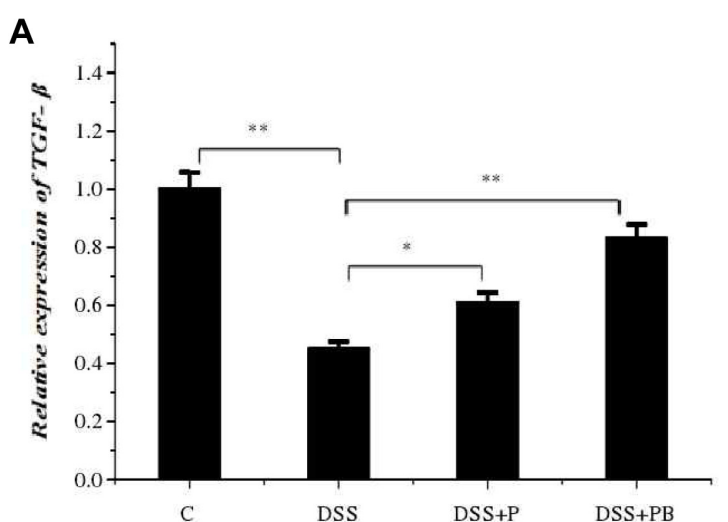

C

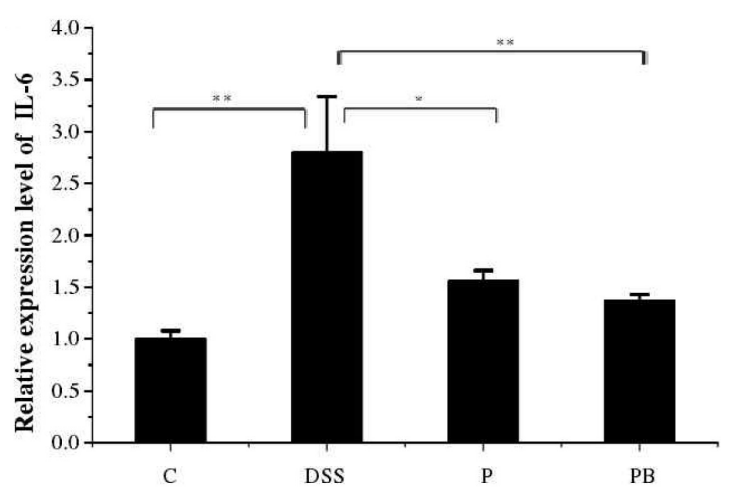

B

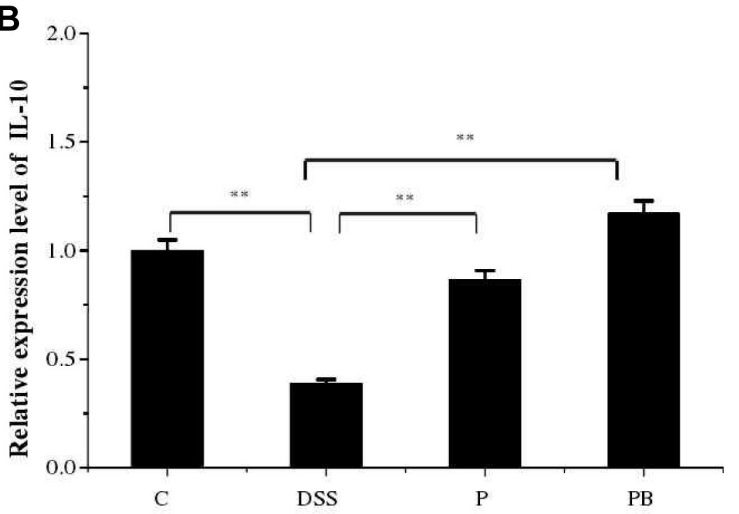

D

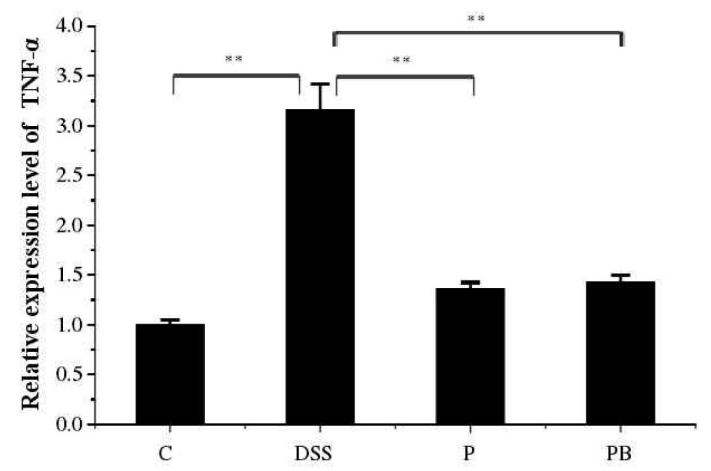

Fig. 3. Effects of probiotics on inflammation marker expression. Relative expression level of TGF- $\beta$ (A); Relative expression level of IL-10 (B); Relative expression level of IL-6 (C); Relative expression level of TNF- $\alpha$ (D). Abbreviation: C, control; DSS: DSStreated group; DSS+P: Probiotic P-treated group; DSS+PB: Probiotic combination-treated group. Values were expressed as the mean $\pm \mathrm{SD},{ }^{*} p<0.05,{ }^{* *} p<0.01$ as conducted.

was enhanced by probiotic B. The results indicate that oral administration of probiotics (P or $\mathrm{PB}$ ) was able to restore the abundances of Bacteriodetes and increase the $\mathrm{B} / \mathrm{F}$ ratio via reshaping of the microbiota composition (Fig. 4B). Biodiversity loss of the intestinal microbial communities in DSS-induced colitis mice has been significantly reduced by probiotic PB treatment.

Furthermore, the relative representative four bacteria (Lactobacillus; Bacteroides, Bifidobacterium bifidum, Escherichia coli) were investigated. The abundance of Escherichia coli and Bacteroides increased significantly in DSS group mice. Of those, the abundance of Escherichia coli increased significantly in DSS group mice, with 200 times as much as the control group (Fig. 4C). On the contrary, the abundances of Bifidobacterium and Lactobacillus decreased significantly in DSS -induced colitis mice (Fig. 4D). Although the Lactobacillus abundances of DSS-induced colitis mice were not significant recovered, the level of Bifidobacterium abundances of DSS-induced colitis mice increased significantly by $95.6 \%$ after one week of probiotic PB treatment.

\section{Effect of Probiotic PB on LPS and Homocysteine Levels of DSS-Induced Colitis Mice}

In order to further evaluate the effect of probiotic PB on the intestinal tract, the concentrations of fecal and plasma endotoxin were investigated. The result showed that the fecal LPS and plasma LPS levels of DSS group were higher than the control group. What's worse, the plasma LPS levels of DSS group were three times that of control group (Fig. 5A). The PLPS/FLPS ratio of DSS group, which was higher than the control group, was down-regulated by probiotics (B or $\mathrm{PB}$ ), however, the difference between probiotic B and probiotic PB was not significant (Fig. 5B). The plasma endotoxin concentration of DSS+PB group was close to that of the control group.

Furthermore, several studies have found that the endothelial cell barrier can be destroyed by homocysteine (Hcy), resulting in increased endothelial cell permeability 
A
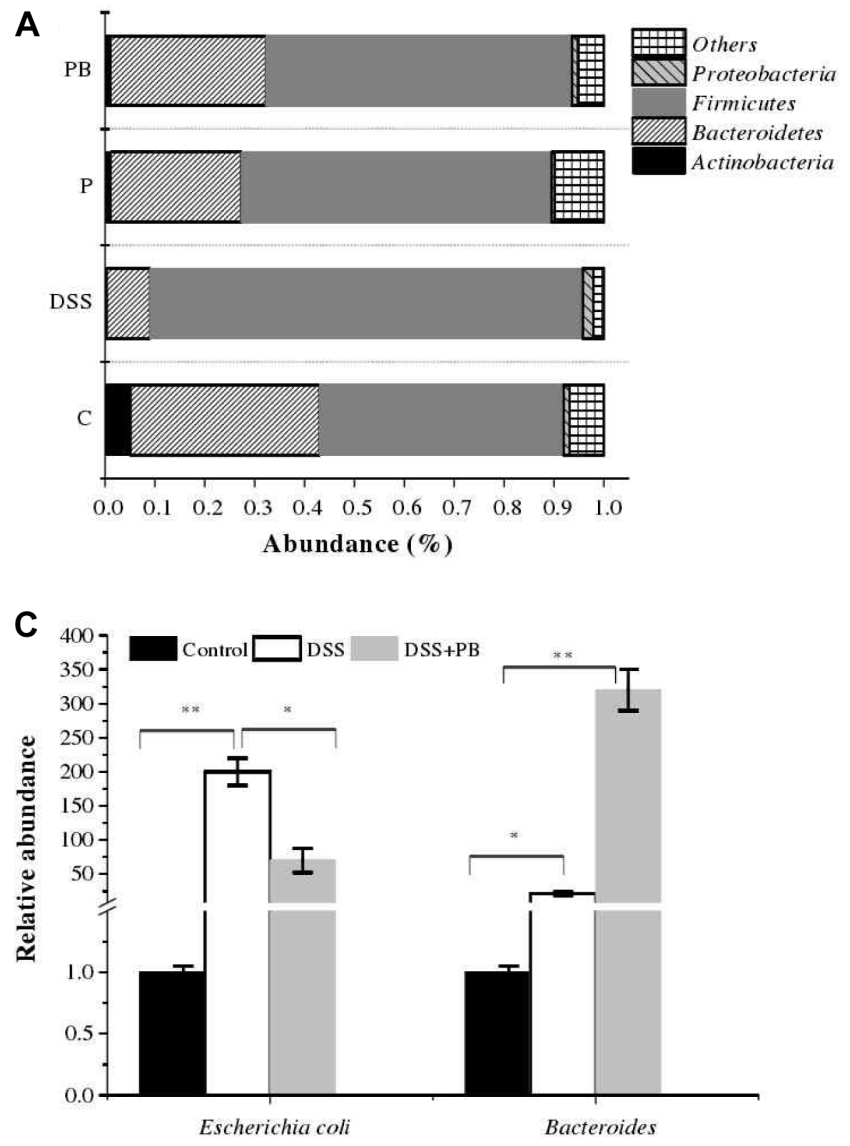

B

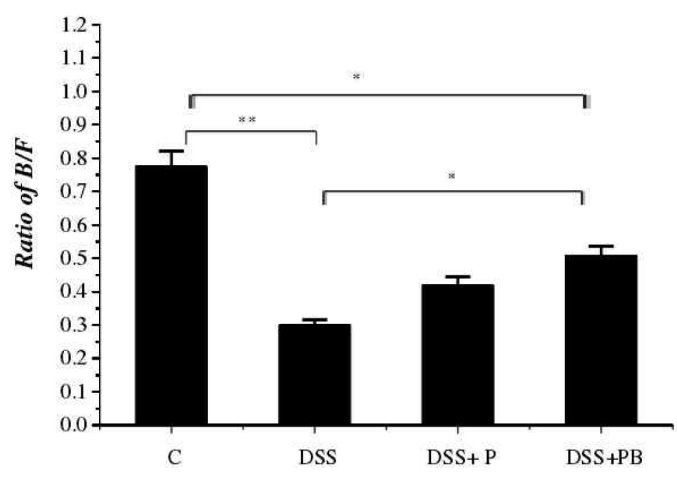

D

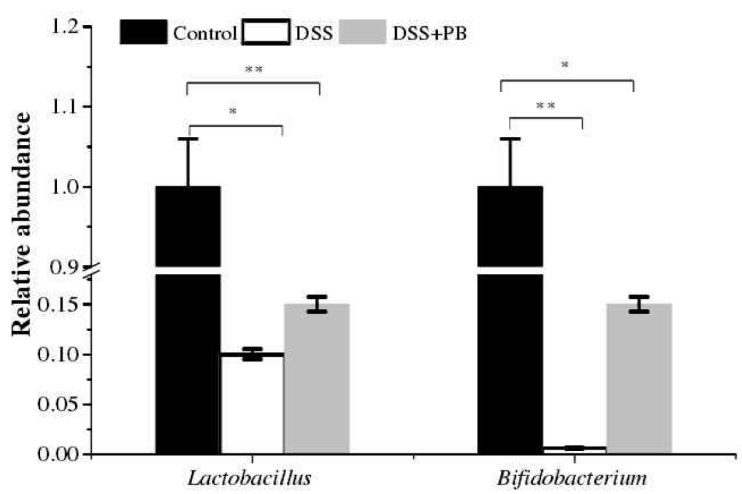

Fig. 4. The abundances of microbial phyla in mice fecal samples (A). In DSS-induced colitis mice and other experimental groups, pooled fecal samples were provided separately. The Bacteriodetes / Firmicutes (B/F) ratio of different groups (B). Alteration of microbiota composition in C57BL/6J mice (C, D). Relative quantity of four representative bacteria was measured by qPCR. Values were expressed as the mean $\pm \mathrm{SD},{ }^{*} p<0.05,{ }^{* *} p<0.01$ as conducted.

A

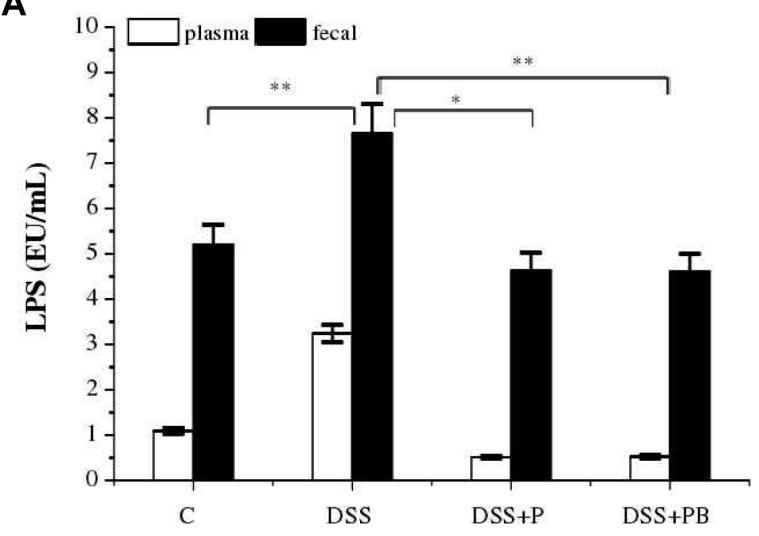

B

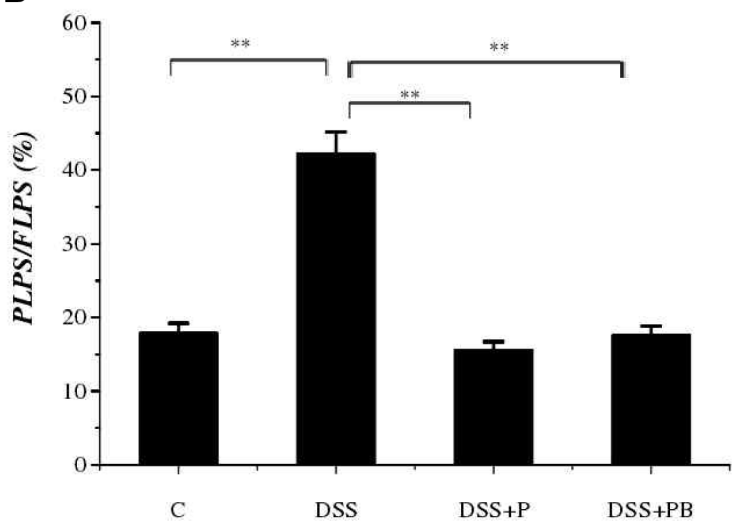

Fig. 5. Effect of probiotic PB on the fecal LPS and plasma LPS levels (A). LAL assay was used to measure the fecal and plasma endotoxin concentrations. All values are indicated as the mean \pm SD. Effect of probiotic PB on the PLPS/ FLPS rate (B). Abbreviation: PLPS, plasma LPS levels; FLPS, fecal LPS levels. Values were expressed as the mean $\pm \mathrm{SD},{ }^{*} p<0.05,{ }^{* *} p<0.01$ as conducted. 
A

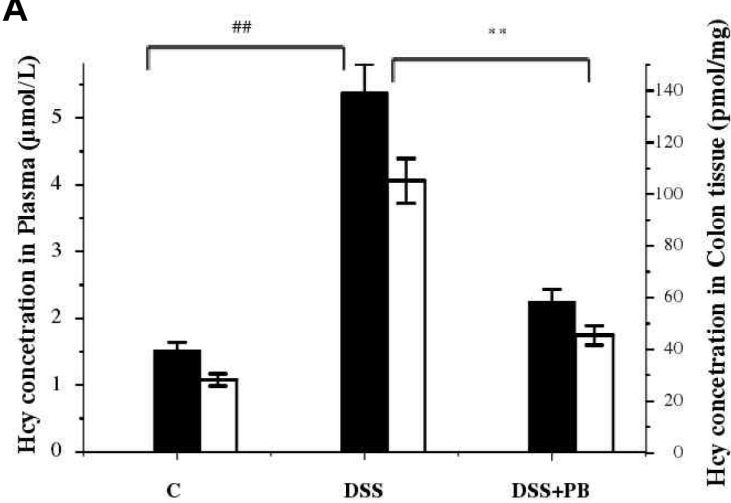

B

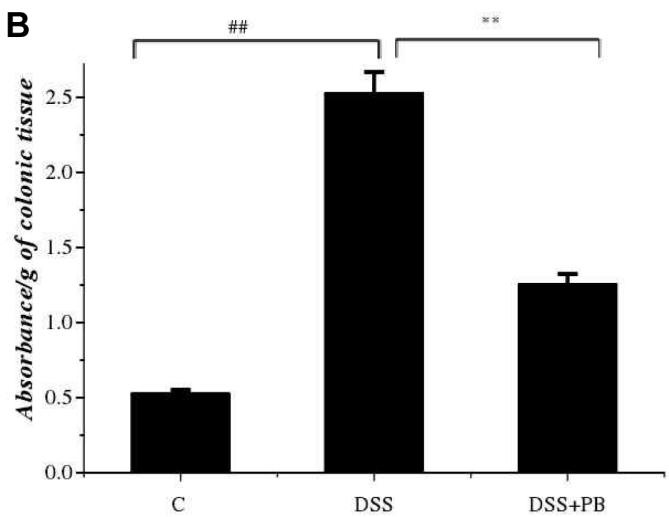

Fig. 6. The levels of homocysteine (Hcy) in plasma and colon mucosa in mice with DSS-induced colitis (A). Abbreviation: C, control; DSS: DSS-treated group; DSS+PB: Probiotic combination-treated group. Effect of probiotic PB on the colonic mucosal permeability of DSS-induced colitis (B). Values were expressed as the mean \pm SD, \#\#p $<0.01$ vs C group, ${ }^{* *} p<0.01$ vs DSS group.

and promoted inflammation [14, 22]. Therefore, the levels of homocysteine in plasma and colon have been investigated. The result showed that the levels of homocysteine in plasma and colon were increased in DSS-induced colitis mice, comparing to the healthy mice (Fig. 6A). As assessed by the Evans blue permeability test, we detected a significant increase in intestinal permeability of DSSinduced colitis mice compared with the healthy mice. Upon administration of $\mathrm{PB}$, there was an approximately 2.01-fold decrease in intestinal permeability of DSS-induced colitis mice (Fig. 6B).

\section{Discussion}

FMT, also called fecal bacteriotherapy with a resolution rate of $83 \%-92 \%$, has been used for 50 years for treatment of Clostridium difcile-associated diarrhea and pseudomembranous colitis with great success and few adverse effects [15]. Developing human gut microbiota has been regarded as a class of therapeutic methods [21, 26, 27]. Therefore, the fecal microorganisms from the fecal flora of healthy adults were selected as the source of probiotics. Strains with high acid and bile salt tolerance were screened to handle the harsh environment of the intestine. Lactobacillus were screened by evaluating the induction capacity on IL-10 (an anti-inflammatory cytokine) production. Bifidobacterium were screened by examining their antimicrobial activity. This method is expected to be used in high-throughput screening of probiotics against DSS-induced colitis or other forms of colitis.

Lactobacillus and Bifidobacteria has shown promising antiinflammatory activity in models of colitis [28, 29], which yielded positive effects in IBD models [30]. Previous studies have shown that numbers of Lactobacilli were significantly lower during the active phase of the disease, and Lactobacillus salivarus, Lactobacillus manihotivorans and Pediococcus acidilactici were present in remission, but not during active inflammation [31]. The present study was performed and showed that Lactobacillus and Bifidobacteria were significantly decreased in DSS-induced colitis. Moreover, the colonic IL- 6 and TNF- $\alpha$ expression was significantly down-regulated and the IL-10 expression was up-regulated in PB-treated mice compared to the DSS group via administration of L. sakei 07 (L07) - B. bifidum B10 combination $(\mathrm{PB})$, resulting in a significant protective effect.

During the last decade, abundant evidence has shown that colonic inflammation is associated with inflammation and gut microbiota [32]. In present study, we found that Bacteroidetes, Actinobacteria decreased and Firmicutes increased in the DSS group mice, significantly. The Bacteriodetes/ Firmicutes (B/F) ratio was increased after oral administration of probiotic PB to DSS-induced colitis mice. Biodiversity of intestinal flora was increased after probiotic PB treatment. In addition, the abundance of Bifidobacterium increased significantly. This result was consistent with the Munyaka $\mathrm{P} \mathrm{M}$ et al. report [33]. It has been reported that microbiota enriched with Bacteroidetes promotes host intestinal immunity and redox responses protecting mice from lethal infectious colitis [6].

Lipopolysaccharide (LPS; endotoxin) produced by gramnegative bacteria increases the permeability of the gut mucosal barrier, increasing LPS translocation into the circulation, augmenting endotoxemia with consequent systemic inflammation [34]. It was found that the plasma 
endotoxin concentrations were decreased by probiotic PB treatment in this study. These results indicate that there are a lot of endotoxins absorbed and accumulated by the intestine rather than excreted out of the body in DSSinduced colitis mice, comparing to the healthy mice. Consequently, the Lactobacillus sakei 07 (L07), Bifidobacterium bifidum B10 and its combination hold potential in UC rehabilitation via immunomodulatory and gut microbiota modulation. Other reports have shown that IL-10 plays a novel role in promoting $\mathrm{H}_{2} \mathrm{~S}$ production and homocysteine metabolism [35]. In the present study the levels of homocysteine in plasma were increased in DSS-induced colitis mice comparing to the healthy mice. This implies that amino acid metabolism is disordered in DSS-induced colitis mice. Therefore, we speculate that the colitis can be relieved by $\mathrm{PB}$ via regulation of the amino acid metabolism.

\section{Conflicts of Interest}

The authors have no financial conflicts of interest to declare.

\section{References}

1. Safarpour AR, Hosseini SV, Mehrabani D. 2013. Epidemiology of inflammatory bowel diseases in iran and Asia; a mini review. Iran. J. Med. Sci. 38: 140-149.

2. Dolan KT, Chang EB. 2016. Diet, gut microbes, and the pathogenesis of inflammatory bowel diseases. Mol. Nutr. Food Res. 61.

3. Frank JA, Reich CI, Sharma S, Weisbaum JS, Wilson BA, Olsen GJ. 2008. Critical evaluation of two primers commonly used for amplification of bacterial 16S rRNA genes. Appl. Environ. Microbiol. 74: 2461-2470.

4. He Q, Li X, Liu C, Su L, Xia Z, Li X, et al. 2016. Dysbiosis of the fecal microbiota in the TNBS-induced Crohn's disease mouse model. Appl. Microbiol. Biotechnol. 100: 4485-4494.

5. Liuyang, Zhao, Xiang, Zhang. 2017. The Composition of colonic commensal bacteria according to anatomical localization in colorectal cancer. Engineering 3: 90-97.

6. Clemente JC, Ursell LK, Parfrey LW, Knight R. 2012. The impact of the gut microbiota on human health: an integrative view. Cell 148: 1258-1270.

7. Cohen RD, Woseth DM, Thisted RA, Hanauer SB. 2000. A meta-analysis and overview of the literature on treatment options for left-sided ulcerative colitis and ulcerative proctitis. Am. J. Gastroenterol. 95: 1263-1276.

8. Ferraris L, Aires J, Waligora-Dupriet AJ, Butel MJ. 2010. New selective medium for selection of bifidobacteria from human feces. Anaerobe 16: 469-471.
9. Plazadíaz J, Fernándezcaballero JÁ, Chueca N, García F, Gómezllorente C, Sáezlara MJ, et al. 2015. Pyrosequencing analysis reveals changes in intestinal microbiota of healthy adults who received a daily dose of immunomodulatory probiotic strains. Nutrients 7: 3999-4015.

10. Kawahara M, Nemoto M, Nakata T, Kondo S, Takahashi H, Kimura B, et al. 2015. Anti-inflammatory properties of fermented soy milk with Lactococcus lactis subsp. lactis SSU2 in murine macrophage RAW264.7 cells and DSSinduced IBD model mice. Int. Immunopharmacol. 26: 295-303.

11. Jo SG, Noh EJ, Lee JY, Kim G, Choi JH, Lee ME, et al. 2016. Lactobacillus curvatus WiKim38 isolated from kimchi induces IL-10 production in dendritic cells and alleviates DSSinduced colitis in mice. J. Microbiol. 54: 503-509.

12. Liu YW, Su YW, Ong WK, Cheng TH, Tsai YC. 2011. Oral administration of Lactobacillus plantarum K68 ameliorates DSS-induced ulcerative colitis in BALB/c mice via the antiinflammatory and immunomodulatory activities. Int. Immunopharmacol. 11: 2159-2166.

13. Scott KP, Martin JC, Duncan SH, Flint HJ. 2014. Prebiotic stimulation of human colonic butyrate-producing bacteria and bifidobacteria, in vitro. Fems Microbiol. Ecol. 87: 30-40.

14. Grimm V, Gleinser M, Neu C, Zhurina D, Riedel CU. 2014. Expression of fluorescent proteins in bifidobacteria for analysis of host-microbe interactions. Appl. Environ. Microbiol. 80: 2842-2850.

15. Palmer R. 2011. Fecal matters. Nat. Med. 17: 150-152.

16. Tyagi N, Moshal KS, Tyagi SC, Lominadze D. 2007. $\gamma$ Aminbuturic acid a receptor mitigates homocysteine-induced endothelial cell permeability. Endothelium 14: 315-323.

17. Chen M, Mei Q, Xu J, Lu C, Fang H, Liu X. 2012. Detection of melatonin and homocysteine simultaneously in ulcerative colitis. Clin. Chim. Acta 413: 30-33.

18. Zijlstra WG, Kampen EJV. 1960. Standardization of hemoglobinometry : I. the extinction coefficient of hemiglobincyanide at $\lambda=540 \mathrm{~m} \mu: \varepsilon 540 \mathrm{HiCN}$. Clin. Chim. Acta 5: 719726.

19. Sang LX, Bing C, Cong D, Nan G, Liu WX, Min J. 2013. Heat-killed VSL\#3 ameliorates dextran sulfate sodium (DSS)-induced acute experimental colitis in rats. Int. J. Mol. Sci. 15: 15-28.

20. Ding SZ, Hao D, Qiao M, Liu XC, Jing HU, Yong-Mei HU, et al. 2016. Effect of homocysteine on the intestinal permeability by regulating MEK-ERK-MLCK signal transduction in experimental colitis rats. Chinese Pharmacological Bulletin 34: 498-502.

21. Tateishi H, Mitsuyama K, Toyonaga A, Tomoyose M, Tanikawa K. 1997. Role of cytokines in experimental colitis: relation to intestinal permeability. Digestion 58: 271-281.

22. Gough E, Shaikh H, Manges AR. 2011. Systematic review of intestinal microbiota transplantation (fecal bacteriotherapy) for recurrent Clostridium difficile infection. Clin. Infect. Dis. 53: $994-1002$. 
23. Guo B, Harstall C, Louie T, Zanten SVV, Dieleman LA. 2012. Systematic review: faecal transplantation for the treatment of Clostridium difficile-associated disease. Aliment. Pharmacol. Ther. 35: 865-875.

24. Khoruts A. 2014. Faecal microbiota transplantation in 2013: developing human gut microbiota as a class of therapeutics. Nat. Rev. Gastroenterol. Hepatol. 11: 79-80.

25. Sokol H, Seksik P, Furet JP, Firmesse O, Nionlarmurier I, Beaugerie L, et al. 2009. Low counts of Faecalibacterium prausnitzii in colitis microbiota. Inflamm. Bowel Dis. 15: 11831189.

26. Uronis JM, Mühlbauer M, Herfarth HH, Rubinas TC, Jones GS, Jobin C. 2009. Modulation of the intestinal microbiota alters colitis-associated colorectal cancer susceptibility. PLoS One. 4: e6026.

27. Scarpellini E, Ianiro G, Attili F, Bassanelli C, Santis AD, Gasbarrini A. 2015. The human gut microbiota and virome: potential therapeutic implications. Dig. Liver Dis. 47: 10071012.

28. Preising J, Philippe D, Gleinser M, Wei H, Blum S, Eikmanns BJ, et al. 2010. Selection of bifidobacteria based on adhesion and anti-inflammatory capacity in vitro for amelioration of murine colitis. Appl. Environ. Microbiol. 76: 3048-3051.

29. Toshimitsu T, Ozaki S, Mochizuki J, Furuichi K, Asami Y. 2017. Effects of Lactobacillus plantarum OLL2712 culture conditions on the anti-inflammatory activity for murine immune cells and obese and type 2 diabetic mice. Appl. Environ. Microbiol. 83: AEM.03001-03016.

30. Prisciandaro L, Geier M, Butler R, Cummins A, Howarth G. 2009. Probiotics and their derivatives as treatments for inflammatory bowel disease. Inflammatory Bowel Diseases 15: 1906-1914.

31. Bullock NR, Booth JC, Gibson GR. 2004. Comparative composition of bacteria in the human intestinal microflora during remission and active ulcerative colitis. Curr. Issues Intest. Microbiol. 5: 59-64.

32. Ghosh S, Dai C, Brown K, Rajendiran E, Makarenko S, Baker J, et al. 2011. Colonic microbiota alters host susceptibility to infectious colitis by modulating inflammation, redox status, and ion transporter gene expression. Am. J. Physiol. Gastrointest Liver Physiol. 301: 39-49.

33. Munyaka PM, Rabbi MF, Khafipour E, Ghia JE. 2016. Acute dextran sulfate sodium (DSS)-induced colitis promotes gut microbial dysbiosis in mice. J. Basic Microbiol. 56: 986-998.

34. Zhang G, Meredith TC, Kahne D. 2013. On the essentiality of lipopolysaccharide to Gram-negative bacteria. Curr. Opinion Microbiol. 16: 779-785.

35. Flannigan KL, Agbor TA, Blackler RW, Kim JJ, Khan WI, Verdu EF, et al. 2014. Impaired hydrogen sulfide synthesis and IL-10 signaling underlie hyperhomocysteinemia-associated exacerbation of colitis. Proc. Natil. Acad. Sci. USA 111: 13559. 